\title{
Only helpful when required: A longevity cost of
}

\section{harbouring defensive symbionts}

C. VORBURGER ${ }^{1 *} \&$ A. GOUSKOV ${ }^{2,3}$

${ }^{1}$ Institute of Integrative Biology, ETH Zürich, 8092 Zürich, Switzerland and EAWAG, Swiss

Federal Institute of Aquatic Science and Technology, 8600 Dübendorf, Switzerland

(christoph.vorburger@eawag.ch)

${ }^{2}$ Institute of Evolutionary Biology and Environmental Studies, University of Zürich, 8057

Zürich, Switzerland

3 present address: EAWAG, Swiss Federal Institute of Aquatic Science and Technology, 8600

Dübendorf, Switzerland (alexandre.gouskov@eawag)

running head: defensive symbionts reduce host longevity

word count: $\quad$ abstract: 140; entire manuscript: 4515; main text: 3400

nr. of references: 32

nr. of figures: $\quad 2$

nr. of tables: $\quad 2$

*correspondence: Christoph Vorburger

EAWAG - ECO

Überlandstrasse $133 \quad \mathrm{Ph}:+41448235196$

8600 Dübendorf $\quad$ Fax: +41448235028

Switzerland christoph.vorburger@eawag.ch

This document is the accepted manuscript version of the following article:

vorburger, C., \& Gouskov, A. (2011). On7y helpful when required: a longevity cost

of harbouring defensive symbionts. Journal of Evolutionary Biology, 24(7),

1611-1617. http://doi.org/10.1111/j.1420-9101.2011.02292.x 


\section{Abstract}

2

3 Maternally transmitted symbionts can spread in host populations if they provide a fitness

4 benefit to their hosts. Hamiltonella defensa, a bacterial endosymbiont of aphids, protects hosts

5 against parasitoids but only occurs at moderate frequencies in most aphid populations. This

6 suggests that harbouring this symbiont is also associated with costs, yet the nature of these

7 costs has remained elusive. Here we demonstrate an important and clearly defined cost:

8 reduced longevity. Experimental infections with six different isolates of $H$. defensa caused

9 strongly reduced lifespans in two different clones of the black bean aphid, Aphis fabae,

10 resulting in a significantly lower lifetime reproduction. However, the two aphid clones were

11 unequally affected by the presence of $H$. defens $a$, and the magnitude of the longevity cost was

12 further determined by genotype $\times$ genotype interactions between host and symbiont, which

13 has important consequences for their coevolution.

14

15 keywords: cost of resistance, longevity, Hamiltonella defensa, parasitoid, symbiosis,

16 trade-off 


\section{Introduction}

Insects and other arthropods are frequently infected with heritable microbial endosymbionts. Such symbionts can increase in frequency in the host population by reproductive manipulations that favour their transmission (e.g. the induction of cytoplasmic incompatibility, feminization, male-killing or parthenogenesis by Wolbachia) (Stouthamer et al., 1999), or by providing a net fitness benefit to their hosts (e.g. Jaenike et al., 2010). Aphids harbour a wide variety of bacterial endosymbionts (Oliver et al., 2010). The obligate endosymbiont Buchnera aphidicola is required for aphid survival and provides a nutritional benefit by synthesizing essential amino acids (Douglas, 1998). In addition to B. aphidicola, aphids commonly harbour facultative or secondary endosymbionts that may be beneficial but are not strictly required for aphid survival. One such symbiont belonging to the Enterobacteriaceae, Hamiltonella defensa (Moran et al., 2005), has been shown to increase aphid resistance to parasitoids (Oliver et al., 2003; Ferrari et al., 2004; Oliver et al., 2005; Vorburger et al., 2009). Symbiont-conferred resistance provides a strong selective advantage in the presence of parasitoids (Herzog et al., 2007; Oliver et al., 2008), yet H. defensa only occurs at low to intermediate frequencies in natural population of aphids (Tsuchida et al., 2002; Simon et al., 2003; Oliver et al., 2006; Vorburger et al., 2009). This suggests that harbouring $H$. defensa also entails costs that select against infected aphids when selection by parasitoids is weak. Indeed, a study on pea aphids, Acyrthosiphon pisum, found that $H$. defensa-infected aphids declined in population cages when competing with uninfected aphids of the same clone in the absence of parasitoids (Oliver et al., 2008). However, the reasons for this decline remained unclear because $H$. defensa had largely positive effects on aphid lifehistory traits: infected lines had significantly shorter generation times and a slightly higher fecundity (Oliver et al., 2008). Comparisons of naturally infected and uninfected clones of the 
black bean aphid, Aphis fabae, also indicated additional benefits rather than costs of possessing $H$. defensa. In a sample of 24 different clones collected in Switzerland, nine were found to harbour $H$. defensa, and they exhibited a higher daily fecundity on average than the 15 clones without $H$. defensa (Vorburger et al., 2009). Similarly, a comparison of life-history traits including a somewhat reduced set of 21 clones of $A$. fabae (seven harbouring $H$. defensa) revealed that adult size and offspring production were higher on average in the H. defensainfected clones (Castañeda et al., 2010). However, these studies only focused on young adults and the correlative evidence from natural infections does not prove a causal link between $H$. defensa infection and aphid fecundity. Thus, identifying the elusive costs of harbouring this defensive symbionts will require the experimental separation of symbiont-conferred effects from genetic variation of the hosts, and a comprehensive assessment of fitness-relevant traits at all life stages. Both is readily possible in aphids. Their clonal mode of reproduction and the possibility to experimentally infect clones with facultative symbionts by microinjection permits the production of sublines with and without symbionts in the same genetic background. We used this approach to introduce six different isolates of $H$. defensa into each of two naturally uninfected clones of A. fabae. A life-table experiment using these lines revealed that infection with $H$. defensa strongly decreased aphid lifespan, resulting in lower lifetime reproduction, and that the magnitude of this longevity cost was determined by genotype $\times$ genotype interactions between host and symbiont.

\section{Material and methods}

\section{Aphid lines}

Aphis fabae is an important pest aphid that is widely distributed in temperate regions of the northern hemisphere. It reproduces by cyclical parthenogenesis, with one sexual, oviparous 
generation over winter followed by many asexual, viviparous generations between spring and autumn. The two clones used in this study, A06-405 and A06-407, were collected during the asexual phase in summer 2006 from the same site in Switzerland. These clones possess different multilocus genotypes based on eight microsatellite loci (Coeur d'Acier et al., 2004), and they were diagnosed as uninfected with facultative endosymbionts by diagnostic PCR (Sandström et al., 2001; Russell et al., 2003; Tsuchida et al., 2006; Vorburger et al., 2009; McLean et al., 2011). Since their collection, they were maintained in the laboratory on broad beans (Vicia faba) under environmental conditions that ensure continued reproduction by apomictic parthenogenesis $\left(16 \mathrm{~h}\right.$ photoperiod at $\left.20^{\circ} \mathrm{C}\right)$. We generated $H$. defensa-infected sublines of these clones using a microinjection protocol as described in Vorburger et al. (2010), transferring symbiont-containing hemolymph from six different clones of A. fabae that were naturally infected with $H$. defensa. Collection details and microsatellite genotypes of the six donor clones as well as the two recipient clones are provided in Table 1 . All of the donor clones exhibit complete or partial resistance to Lysiphlebus fabarum, the most important parasitoid of A. fabae (Vorburger et al., 2009; R. Rouchet \& C. Vorburger, unpublished data).

Based on a combination of diagnostic PCR and of sequencing the amplicons of PCR reactions using the general bacterial primers $10 \mathrm{~F}$ and $35 \mathrm{R}$ for the $16 \mathrm{~S}$ ribosomal RNA gene (Sandström et al., 2001; Russell \& Moran, 2005), H. defensa was the only facultative endosymbiont present in the donor clones. The six H. defensa isolates are labelled H 9, H 30; H 76, H 323, H 402 and H Af6, in reference to their clone of origin. Although the different donor clones were collected from as far apart as southern France and Switzerland (Table 1), they should not be regarded as coming from different, isolated populations. Aphids have a high dispersal ability (Llewellyn et al., 2003), and a population genetic survey using microsatellites found very low levels of genetic differentiation in A. fabae across Europe (Sandrock et al., 
submitted). We have no genetic information about the relatedness among the $H$. defensa isolates used here and their relatedness to known defensive isolates in other aphid species (e.g. Oliver et al., 2005), but phylogenetic analyses suggest that horizontal transmission among species occurs at least occasionally (Sandström et al., 2001; Russell et al., 2003).

Successful transmission of $H$. defensa by microinjection normally results in stable infections of clonal lines, since vertical transmission under laboratory conditions is virtually perfect. We confirmed the presence of $H$. defensa in the recipient lines by diagnostic PCR for the first three generations after transfection as well as immediately before use in the experiment. For one of the two recipient clones (A06-407) we also verified that protection against parasitoids by $H$. defensa is still expressed in the new genetic background (R. Rouchet \& C. Vorburger, unpublished data). The tranfected lines carried their $H$. defens $a$ infections for between 20 and 40 generations prior to the experiment described below.

\section{Life-table experiment}

To estimate potential effects of the infection with $H$. defens $a$ on aphid life-history traits we carried out a life-table experiment similar to the one described in Vorburger (2005). The experiment took place in a climatised room under fluorescent light with a $16 \mathrm{~h}$ photoperiod at $20^{\circ} \mathrm{C}$. All 14 aphid lines (two uninfected and six infected from each clone) were split into eight replicates that were maintained on caged $V$. faba seedlings growing in plastic pots of 0.071 volume. One replicate per line was assigned to a random position in eight different plastic trays (randomised complete blocks). To avoid the potential inflation of among-line variation by maternal or grand-maternal environmental effects carried over from the stock culture, we maintained the replicates for two generations (each generation on a fresh plant) before we assayed the life-history traits in the third generation after the split. The test generation was initiated by placing four adult females from the second generation on a new 
118 seedling, allowing them to reproduce for $4 \mathrm{~h}$, and then removing the adults and all but one

119 newborn nymph from the plant. These individuals represented the experimental cohort, which

120 was checked daily for survival. After six days, we started monitoring the animals every $8 \mathrm{~h}$ to

121 determine the time of their final moult (adult ecdysis), from which we calculated development

122 time (duration from birth to adult ecdysis). We weighed all newly moulted adults to the

123 nearest $\mu \mathrm{g}$ on a Mettler MX5 microbalance (Mettler Toledo GmbH, Greifensee, Switzerland)

124 to determine their fresh mass as an estimate of body size and then replaced them on their

125 plants. After that, their offspring were removed and counted daily until they died. To ensure

126 that the aphids developed under favourable conditions and that they remained easy to find

127 every day, we transferred the adults to new seedlings every 5 days. From the number of

128 offspring produced over the first 7 days of reproduction we calculated the daily fecundity

129 (mean number of offspring produced per day) as an estimate of reproductive performance of

130 young adults. This estimate could not be obtained for individuals that survived for less than 7

131 days after adult ecdysis, which was then treated as missing data. We also determined the

132 lifetime reproductive output (total number of offspring produced from adult ecdysis until

133 death) and the age at death. Finally, to obtain an overall fitness estimate for each individual,

134 we used the complete life-table data to calculate $F^{\prime}$, following Service \& Lenski (1982):

$$
F_{i}^{\prime}=\sum_{x=0}^{\infty} F_{N}^{-x} S_{x i} B_{x i}
$$

where $S_{x i}$ is the survival of individual $i$ to age class $x$ (one or zero), $B_{x i}$ is the number of daughters produced by individual $i$ in age class $x$, and $F_{N}$ is the finite rate of increase of the

140 entire experimental cohort over the duration of one age class (i.e. 1 day in this experiment).

$141 \quad F_{N}$ is obtainable from the stable-age equation (Lenski \& Service, 1982, equ. 4), which we

142 solved iteratively. We found $F_{N}$ to be 1.37 , which corresponds to the mean of the $F_{i}^{\prime}$ (Lenski 
$143 \&$ Service, 1982). $F_{i}^{\prime}$ is generally interpreted as the lifetime contribution of individual $i$ to

144 population growth, which is a useful measure of individual fitness (Lenski \& Service, 1982).

145 Two individuals were accidentally killed in a transfer during the experiment and had to be 146 excluded from all analyses.

\section{Statistical analyses}

149 Aphid life-history traits were analysed with general linear models using the open source 150 statistical software R 2.9.2 (R Development Core Team, 2009). We tested for the effects of 151 experimental block, aphid clone, subline and the clone $\times$ subline interaction. The variance

152 among sublines and the variance explained by the clone $\times$ subline interaction was further 153 partitioned into contributions from the variance between uninfected $(\mathrm{H}-)$ and infected $(\mathrm{H}+)$ 154 sublines and the variance within $\mathrm{H}+$ sublines (i.e. among different $H$. defensa isolates), using 155 linear orthogonal contrasts. Because the block effect was far from significant in all analyses,

156 we pooled the variance among blocks into the residual term. Survival data were analysed with 157 a Cox proportional hazards regression, testing for the effects of aphid clone, subline and their 158 interaction.

160 Results

Infection with $H$. defensa had no detectable effect on aphid development time, but development was significantly slower in clone A06-407 than in clone A06-405 (Table 2, Fig. 1a). Aphid body size measured as adult fresh mass did not differ significantly between clones, nor was there a significant difference among sublines, but the contrast between the means of

166 uninfected (H-) and infected (H+) sublines indicated a slight but significant reduction of body

167 size in the presence of $H$. defensa (Table 2, Fig. 1b). The fecundity of young adult aphids was 
168 similar for both clones but exhibited variation among sublines (Table 2, Fig. 1c). The

169 marginally significant contrast between $\mathrm{H}$ - and $\mathrm{H}+$ suggests that this was at least partly due to 170 a slight reduction in fecundity of infected aphids. However, this effect differed between the 171 two aphid clones as indicated by the significant aphid clone $\times$ subline interaction (Table 2 ), 172 which largely reflected the inconsistent effects of the different isolates of $H$. defensa on the 173 two clones (Table 2, Fig. 1c).

174 The only really striking effect of harbouring $H$. defensa we observed was a reduction in 175 longevity. An inspection of the survivorship curves (Fig. 2) shows clearly that in both clones, 176 mortality rates differed among sublines, with uninfected aphids living longer on average than 177 aphids harbouring $H$. defensa. This resulted in a significant subline effect in a Cox 178 proportional hazards regression $\left(\mathrm{LR} \chi^{2}=78.1 \mathrm{df}=6, P<0.001\right)$. Interestingly, the two 179 clones were unequally affected by the presence of $H$. defensa (clone $\times$ subline interaction, LR $180 \chi^{2}=38.3$, df $\left.=6, P<0.001\right)$. The reduction in longevity by the different isolates of $H$. defensa 181 was much more severe in clone A06-407 than in A06-405 (Fig. 2). This is also evident when 182 survivorship is analysed as age at death (Fig. 1d). Individuals of both clones died younger 183 when they were infected with $H$. defensa. This seems to be a rather general effect of this symbiont because the contrast analysis showed that the significant subline effect was largely due to the difference between $\mathrm{H}-$ and $\mathrm{H}+$ (Table 2). However, clone A06-407, which produced longer-lived individuals when uninfected (44.0 days $\pm 1.3 \mathrm{SE}$ vs. $32.3 \pm 3.9$ days in 187 A06-405), suffered a reduction by almost two thirds to $16.5 \pm 0.9$ days average lifespan, whereas A06-405 only suffered a reduction of about one fourth to $23.6 \pm 1.0$ days average

189 lifespan, reversing the order of their performance in the presence of $H$. defensa (Fig. 1d). This 190 was reflected in the significant clone $\times$ subline interaction on the age at death, to which the 191 contrast between $\mathrm{H}$ - and $\mathrm{H}+$ contributed most of the variation. Yet the contrast analysis also 
192 showed that the effects of the different isolates of $H$. defensa on longevity depended on the 193 aphid clone (Table 2).

194 The marked differences in longevity we observed translated directly into differences in the 195 most inclusive fitness estimates we obtained, namely the lifetime reproductive output and $F_{i}{ }^{\prime}$, 196 a life-table based measure of an individual's contribution to population growth, of which the 197 means can be interpreted as an estimate of the finite rate of increase for each subline (Lenski

198 \& Service, 1982; Service \& Lenski, 1982). Both measures varied significantly among

199 sublines (Table 2, Figs. 1e, f), and the contrast between $\mathrm{H}-$ and $\mathrm{H}+$ explained much of this

200 variation (Table 2). In accordance with the stronger reduction of longevity, the negative effect 201 on fitness was more pronounced in clone A06-407. However, different isolates of $H$. defensa 202 contributed unequally to this fitness reduction: clone A06-407 suffered most from the 203 presence of isolates $\mathrm{H} 30$ and $\mathrm{H}$ 76, for example. This was supported by a significant clone $\times$ 204 subline interaction for both traits, much of which is explained by the interaction of the 205 different isolates within the $\mathrm{H}+$ group and the two aphid clones (Table 2).

\section{Discussion}

By demonstrating substantial fitness costs of harbouring $H$. defensa, this experiment supports the notion that infected aphids are competitively inferior in the absence of parasitoids

211 (Oliver et al., 2008), thus preventing the fixation of this facultative symbiont in natural 212 populations. Our study is the first to provide a mechanistic understanding of these costs: infection with $H$. defensa shortens an aphid's life. The shorter lifespan was the main reason

214 for the reduced lifetime reproduction of infected aphids in our experiment, because the 215 observed reductions of fecundity were small. Under the benign conditions of our laboratory 216 experiment, the costs of harbouring $H$. defensa in terms of lifetime reproductive output were 
217 quite substantial (Fig. 1e), yet this result should be interpreted with caution. In the field, 218 aphids are unlikely to live their full potential lifespans due to extrinsic sources of mortality 219 such as predation, and in periods of populations growth (e.g. the exponential growth phase of 220 aphid populations in spring), early reproduction contributes more to fitness than late 221 reproduction (Stearns, 1992). Therefore, the fitness costs of harbournig $H$. defensa may be 222 less pronounced under field conditions.

It is tempting to conclude that the reduction of longevity caused by H. defensa is mechanistically linked to the protection it provides against parasitoids. The protection results from the presence of toxin-encoding bacteriophages within $H$. defensa's genome (Degnan \& Moran, 2008a, b; Oliver et al., 2009). These toxins appear to kill the eggs or early larval stages of parasitoids, but they may also have negative effects on the aphids themselves, thus reducing their longevity. This hypothesis is yet to be tested. It will be particularly important to know whether the toxin genes are expressed constitutively or only upon attack by parasitoids. early mortality, it also showed that the magnitude of these costs depends on the host's genetic 232 background. One aphid clone was much more affected than the other. Furthermore, the costs 233 depended on the exact combination of host clone and symbiont isolate, reflecting a genotype $\times$ genotype interaction between $A$. fabae and $H$. defensa. This may have important consequences for the frequencies of $H$. defensa in natural aphid populations as well as for the dynamics of host-symbiont coevolution. It suggests that the cost-benefit ratio of possessing $H$.

237 defensa would differ among aphid genotypes. For certain host-symbiont combinations (e.g. 238 aphid clone A06-407 with $H$. defensa isolate H 30 in the present experiment; Figs. 1 \& 2), the 239 net effect on aphid fitness resulting from the symbiosis is likely to be negative, despite 240 increased resistance to parasitoids. Such combinations are unlikely to be encountered in the 241 field. In other combinations (e.g. clone A06-405 with H76), the benefits of increased 
resistance are likely to exceed the longevity cost. Obviously, the cost-benefit ratio will also be

243 affected by the risk of attack by parasitoids, which will vary in space as well as in time. We

244 can thus expect that the $H$. defensa-infected aphids we observe in the field do not represent

245 random combinations of host and symbiont genotypes, but rather well-matching combinations

246 that were favoured by natural selection because the protective effect of $H$. defensa comes at

247 comparatively low costs. A potential test of this hypothesis would include a similar

248 experiment using lines from which natural infections with $H$. defensa were removed by

249 antibiotic curing (e.g. McLean et al., 2011), the prediction being that the gain in longevity

250 would then be relatively modest. If this was indeed the case, it would help explain why

251 comparisons of naturally infected and uninfected clones of A. fabae did not reveal any

252 evidence for costs (Vorburger et al., 2009; Castañeda et al., 2010). Another explanation could

253 be that just like other vertically transmitted symbionts, $H$. defensa relies on host reproduction

254 for its own transmission. That is why its ability to protect aphids against parasitoids evolved

255 in the first place, but the same would apply to the symbiont's own effects on host survival.

256 Upon successful infection of a host lineage, $H$. defensa should evolve to be less 'virulent' in

257 that it shows reduced effects on host survival. However, evolution of reduced virulence

258 towards the host could be constrained if it entailed reduced protection against parasitoids.

259 Whether such a trade-off exists remains to be investigated.

260 To conclude, we show that in the black bean aphid, A. fabae, the defensive symbiont $H$.

261 defensa is only helpful when required, i.e. when aphids are under strong selection by

262 parasitoids. In the absence of parasitoids, harbouring $H$. defensa is associated with costs

263 which are mostly due to a reduction of host longevity. The magnitude of the negative effect

264 on host survival is to a large extent determined by genotype $\times$ genotype interactions between

265 hosts and symbionts, which has important consequences for their coevolution. 


\section{Acknowledgements}

We thank Daniel Bopp, Paula Rodriguez and Romain Rouchet for help with microinjections,

Julia Ferrari for help with endosymbiont screening, and Luis Cayetano for comments on the manuscript. This work was supported by the Swiss National Science Foundation (SNSF).

\section{References}

Castañeda, L.E., Sandrock, C. \& Vorburger, C. 2010. Variation and covariation of life history traits in aphids are related to infection with the facultative bacterial endosymbiont Hamiltonella defensa. Biol. J. Linn. Soc. 100: 237-247.

Coeur d'Acier, A., Sembene, M., Audiot, P. \& Rasplus, J.Y. 2004. Polymorphic microsatellites loci in the black Aphid, Aphis fabae Scopoli, 1763 (Hemiptera, Aphididae). Mol. Ecol. Notes. 4: 306-308.

Degnan, P.H. \& Moran, N.A. 2008a. Diverse phage-encoded toxins in a protective insect endosymbiont. Appl. Environ. Microbiol. 74: 6782-6791.

Degnan, P.H. \& Moran, N.A. 2008b. Evolutionary genetics of a defensive facultative symbiont of insects: exchange of toxin-encoding bacteriophage. Mol. Ecol. 17: 916-929.

Douglas, A.E. 1998. Nutritional interactions in insect-microbial symbioses: Aphids and their symbiotic bacteria Buchnera. Annu. Rev. Entomol. 43: 17-37.

Ferrari, J., Darby, A.C., Daniell, T.J., Godfray, H.C.J. \& Douglas, A.E. 2004. Linking the bacterial community in pea aphids with host-plant use and natural enemy resistance. Ecol. Entomol. 29: 60-65.

Herzog, J., Müller, C.B. \& Vorburger, C. 2007. Strong parasitoid-mediated selection in experimental populations of aphids. Biol. Lett. 3: 667-669. symbiosis: recent spread of a Drosophila defensive symbiont. Science 329: 212-215. calculated from schedules of survivorship and fecundity. Ecology 63: 655-662. 
Llewellyn, K.S., Loxdale, H.D., Harrington, R., Brookes, C.P., Clark, S.J. \& Sunnucks, P. 2003. Migration and genetic structure of the grain aphid (Sitobion avenae) in Britain related to climate and clonal fluctuation as revealed using microsatellites. Mol. Ecol. 12: 21-34.

McLean, A.H.C., van Asch, M., Ferrari, J. \& Godfray, H.C.J. 2011. Effects of bacterial secondary symbionts on host plant use in pea aphids. Proc. R. Soc. Lond. B 278: 760-766.

Moran, N.A., Russell, J.A., Koga, R. \& Fukatsu, T. 2005. Evolutionary relationships of three new species of Enterobacteriaceae living as symbionts of aphids and other insects. Appl. Environ. Microbiol. 71: 3302-3310.

Oliver, K.M., Russell, J.A., Moran, N.A. \& Hunter, M.S. 2003. Facultative bacterial symbionts in aphids confer resistance to parasitic wasps. Proc. Natl. Acad. Sci. U. S. A. 100: $1803-1807$.

Oliver, K.M., Moran, N.A. \& Hunter, M.S. 2005. Variation in resistance to parasitism in aphids is due to symbionts not host genotype. Proc. Natl. Acad. Sci. U. S. A. 102: 1279512800.

Oliver, K.M., Moran, N.A. \& Hunter, M.S. 2006. Costs and benefits of a superinfection of facultative symbionts in aphids. Proc. R. Soc. Lond. B 273: 1273-1280.

Oliver, K.M., Campos, J., Moran, N.A. \& Hunter, M.S. 2008. Population dynamics of defensive symbionts in aphids. Proc. R. Soc. Lond. B 275: 293-299.

Oliver, K.M., Degnan, P.H., Hunter, M.S. \& Moran, N.A. 2009. Bacteriophages encode factors required for protection in a symbiotic mutualism. Science 325: 992-994.

Oliver, K.M., Degnan, P.H., Burke, G.R. \& Moran, N.A. 2010. Facultative symbionts in aphids and the horizontal transfer of ecologically important traits. Annu. Rev. Entomol. 55: 247-266.

R Development Core Team 2009. R: a language and environment for statistical computing. R Foundation for Statistical Computing, Vienna, Austria. ISBN 3-900051-07-0, URL http://www.R-project.org.

Russell, J.A., Latorre, A., Sabater-Muñoz, B., Moya, A. \& Moran, N.A. 2003. Side-stepping secondary symbionts: widespread horizontal transfer across and beyond the Aphidoidea. Mol. Ecol. 12: 1061-1075.

Russell, J.A. \& Moran, N.A. 2005. Horizontal transfer of bacterial symbionts: Heritability and fitness effects in a novel aphid host. Appl. Environ. Microbiol. 71: 7987-7994.

Sandrock, C., Razmjou, J. \& Vorburger, C. submitted. Climate effects on life cycle variation and population genetic architecture of the black bean aphid, Aphis fabae. 
331

332

333

334

335

336

337

338

339

340

341

342

343

344

345

346

347

348

349

350

351

352

353

354

355 356

Sandström, J.P., Russell, J.A., White, J.P. \& Moran, N.A. 2001. Independent origins and horizontal transfer of bacterial symbionts of aphids. Mol. Ecol. 10: 217-228.

Service, P.M. \& Lenski, R.E. 1982. Aphid genotypes, plant phenotypes, and genetic diversity: a demographic analysis of experimental data. Evolution. 36: 1276-1282.

Simon, J.C., Carre, S., Boutin, M., Prunier-Leterme, N., Sabater-Munoz, B., Latorre, A. \& Bournoville, R. 2003. Host-based divergence in populations of the pea aphid: insights from nuclear markers and the prevalence of facultative symbionts. Proc. R. Soc. Lond. B 270: 1703-1712.

Stearns, S.C. 1992. The Evolution of Life Histories. Oxford University Press, New York.

Stouthamer, R., Breeuwer, J.A.J. \& Hurst, G.D.D. 1999. Wolbachia pipientis: Microbial manipulator of arthropod reproduction. Annu. Rev. Microbiol. 53: 71-102.

Tsuchida, T., Koga, R., Shibao, H., Matsumoto, T. \& Fukatsu, T. 2002. Diversity and geographic distribution of secondary endosymbiotic bacteria in natural populations of the pea aphid, Acyrthosiphon pisum. Mol. Ecol. 11: 2123-2135.

Tsuchida, T., Koga, R., Sakurai, M. \& Fukatsu, T. 2006. Facultative bacterial endosymbionts of three aphid species, Aphis craccivora, Megoura crassicauda and Acyrthosiphon pisum, sympatrically found on the same host plants. Appl. Entomol. Zool. 41: 129-137.

Vorburger, C. 2005. Positive genetic correlations among major life-history traits related to ecological success in the aphid Myzus persicae. Evolution. 59: 1006-1015.

Vorburger, C., Sandrock, C., Gouskov, A., Castañeda, L.E. \& Ferrari, J. 2009. Genotypic variation and the role of defensive endosymbionts in an all-parthenogenetic hostparasitoid interaction. Evolution. 63: 1439-1450.

Vorburger, C., Gehrer, L. \& Rodriguez, P. 2010. A strain of the bacterial symbiont Regiella insecticola protects aphids against parasitoids. Biol. Lett. 6: 109-111. 


\section{$357 \quad$ Figure captions}

358

359 Fig. 1 Life-history traits of infected and uninfected sublines of two clones of Aphis fabae:

360 development time from birth to adult ecdysis (a), adult mass measured as fresh weight of

361 newly ecdysed adults (b), daily fecundity averaged over the first 7 days of reproduction (c),

362 age at death (d), lifetime number of offspring (e), and overall fitness measured as $F_{i}{ }^{\prime}$, an

363 estimate of individual contribution to population growth (f). The bars left of the gap contrast

364 the means (+ 1 SE) of the uninfected and all infected sublines, the bars right of the gap depict

365 the means of infected sublines separately for each isolate of Hamiltonella defensa.

367 Fig. 2 Survivorship curves of sublines of Aphis fabae clone A06-405 (a) and A06-407 (b),

368 that are either uninfected (black lines) or experimentally infected with one of six different

369 isolates of the defensive endosymbiont Hamiltonella defensa (coloured lines). 
Table 1 Collection information and genotypes at eight microsatellite loci (Coeur d'Acier et al., 2004) for the eight clones of Aphis fabae used in this study. The two recipient clones used in the life-table experiment were experimentally infected with Hamiltonella defensa by microinjection of hemolymph from each of the six donor clones.

\begin{tabular}{|c|c|c|c|c|c|c|c|c|c|c|c|c|}
\hline \multirow[b]{2}{*}{ Sample ID } & \multirow[b]{2}{*}{ Collection site } & \multirow[b]{2}{*}{ Collection date } & \multirow[b]{2}{*}{ Host plant } & \multirow[b]{2}{*}{ Facultative symbiont } & \multicolumn{8}{|c|}{ Microsatellite locus } \\
\hline & & & & & AF-48 & AF-50 & AF-82 & AF-85 & AF-86 & AF-181 & AF-beta & AF-F \\
\hline \multicolumn{13}{|c|}{ Recipient clones } \\
\hline A06-405 & St. Margrethen, Switzerland & 01.07 .2006 & Chenopodium album & - & 315317 & 257257 & 167177 & 220220 & 217219 & 311311 & 280282 & 127127 \\
\hline A06-407 & St. Margrethen, Switzerland & 01.07 .2006 & Chenopodium album & - & 315315 & 272272 & 177177 & 218220 & 215215 & 309309 & 280282 & 127127 \\
\hline \multicolumn{13}{|c|}{ Donor clones } \\
\hline A06-9 & La Spezia, Italy & 08.05 .2006 & Vicia faba & Hamiltonella defensa & 315321 & 257272 & 171177 & 220222 & 219219 & 309309 & 280282 & 127127 \\
\hline A06-30 & Sarzana, Italy & 08.05 .2006 & Vicia faba & Hamiltonella defensa & 315315 & 257257 & 177177 & 220220 & 219219 & 311311 & 266282 & 132136 \\
\hline A06-76 & La Grande Motte, France & 17.05.2006 & Chenopodium album & Hamiltonella defensa & 315315 & 257272 & 192204 & 220222 & 217219 & 311313 & 280280 & 127127 \\
\hline A06-323 & Aesch, Switzerland & 27.06 .2006 & Vicia faba & Hamiltonella defensa & 315315 & 257272 & 177177 & 220222 & 215215 & 309309 & 280280 & 134136 \\
\hline A06-402 & St. Margrethen, Switzerland & 01.07 .2006 & Chenopodium album & Hamiltonella defensa & 315315 & 257257 & 177177 & 220220 & 219219 & 309313 & 280280 & 127127 \\
\hline Af6 & Zürich, Switzerland & 25.05 .2004 & Euonymus europaeus & Hamiltonella defensa & 315315 & 257257 & 177177 & 218222 & 219219 & 311317 & 280282 & 127134 \\
\hline
\end{tabular}


Table 2 General linear model results for the six life-history traits measured.

\begin{tabular}{lrlll}
\hline Source of variation & df & MS & $\boldsymbol{F}$ & $\boldsymbol{P}$ \\
\hline $\begin{array}{l}\text { Development time } \\
\text { Aphid clone }\end{array}$ & 1 & 0.509 & 4.796 & 0.03 \\
Subline & 6 & 0.126 & 1.190 & 0.319 \\
$\quad$ between H- and H+ & 1 & 0.188 & 1.777 & 0.186 \\
among H+ & 5 & 0.114 & 1.076 & 0.379 \\
Aphid clone $\times$ subline & 6 & 1.272 & 1.200 & 0.314 \\
between H- and H+ & 1 & 0.022 & 0.204 & 0.653 \\
$\quad$ among H+ & 5 & 0.148 & 1.395 & 0.234 \\
Residual & 89 & 0.106 & &
\end{tabular}

\section{Adult mass}

$\begin{array}{lllll}\text { Aphid clone } & 1 & 0.017 & 1.194 & 0.278 \\ \text { Subline } & 6 & 0.022 & 1.561 & 0.168 \\ \text { between H- and H+ } & 1 & 0.080 & 5.639 & 0.020 \\ \text { among H+ } & 5 & 0.011 & 0.763 & 0.579 \\ \text { Aphid clone × subline } & 6 & 0.013 & 0.903 & 0.496 \\ \text { between H- and H+ } & 1 & 0.028 & 1.977 & 0.163 \\ \text { among H+ } & 5 & 0.010 & 0.668 & 0.649 \\ \text { Residual } & 89 & 0.014 & & \end{array}$

Daily fecundity

$\begin{array}{lcccc}\text { Aphid clone } & 1 & 1.441 & 2.409 & 0.125 \\ \text { Subline } & 6 & 1.378 & 2.304 & 0.043 \\ \text { between H- and H+ } & 1 & 2.425 & 4.055 & 0.048 \\ \text { among H+ } & 5 & 1.185 & 1.982 & 0.092 \\ \text { Aphid clone × subline } & 6 & 1.772 & 2.964 & 0.012 \\ \text { between H- and H+ } & 1 & 0.608 & 1.017 & 0.317 \\ \text { among H+ } & 5 & 1.988 & 3.324 & 0.009 \\ \text { Residual } & 71 & 0.598 & & \end{array}$

Table 1 continues on next page 
Table 1 continued

Age at death

Aphid clone

Subline

643.2

15.050

$<0.001$

between $\mathrm{H}-$ and $\mathrm{H}+$

725.9

16.984

$<0.001$

among $\mathrm{H}+$

3981.7

93.248

$<0.001$

Aphid clone $\times$ subline

75.86

1.777

0.125

between $\mathrm{H}$ - and $\mathrm{H}+$

315.2

7.375

$<0.001$

among $\mathrm{H}+$

1143.3

26.775

$<0.001$

3.478

0.006

Residual

96

42.7

\section{Lifetime reproduction}

\begin{tabular}{lrrrr} 
Aphid clone & 1 & 7677.8 & 23.663 & $<0.001$ \\
Subline & 6 & 1578.5 & 4.865 & $<0.001$ \\
$\quad$ between H- and H+ & 1 & 6004.0 & 18.502 & $<0.001$ \\
among H+ & 5 & 692.1 & 2.133 & 0.068 \\
Aphid clone $\times$ subline & 6 & 1591.4 & 4.905 & $<0.001$ \\
$\quad$ between H- and H+ & 1 & 1895.0 & 5.840 & 0.018 \\
among H+ & 5 & 1532.2 & 4.722 & $<0.001$ \\
Residual & 96 & 324.5 & & \\
& & & & 0.004 \\
$F_{i}{ }^{\prime}$ (finite rate of increase) & & & & $<0.001$ \\
Aphid clone & 1 & 2.220 & 8.594 & $<0.001$ \\
Subline & 6 & 1.373 & 5.314 & 0.020 \\
between H- and H+ & 1 & 4.597 & 17.790 & 0.007 \\
among H+ & 5 & 0.728 & 2.816 & 0.546 \\
Aphid clone $\times$ subline & 6 & 0.825 & 3.194 & 0.004 \\
between H- and H+ & 1 & 0.095 & 0.368 & 3.761 \\
among H+ & 5 & 0.972 & & \\
Residual & 96 & 0.258 & & \\
\hline
\end{tabular}



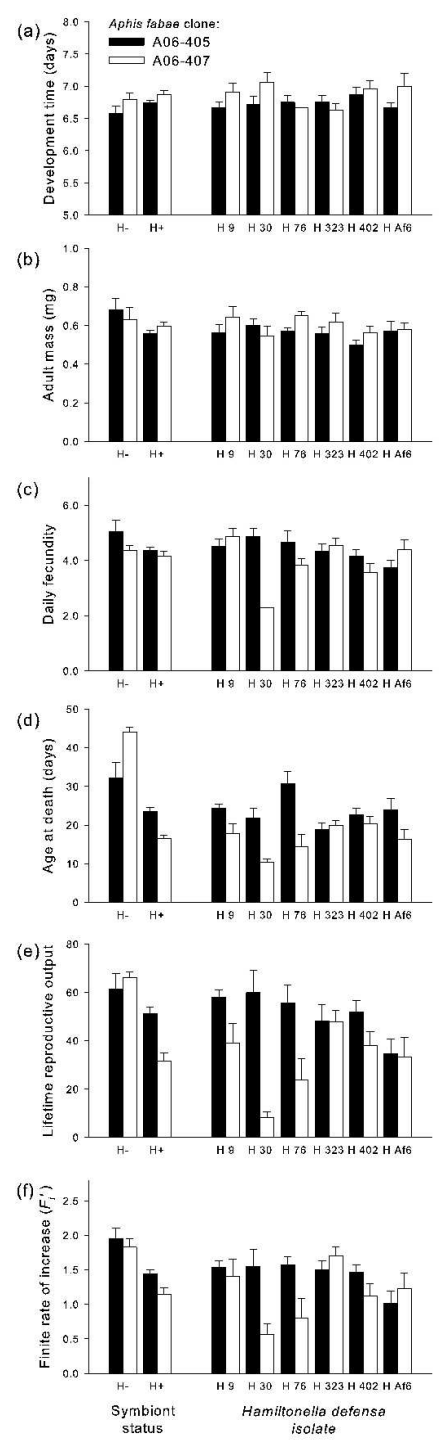

$168 \times 567 \mathrm{~mm}(600 \times 600 \mathrm{DPI})$ 
(a) Clone A06-405

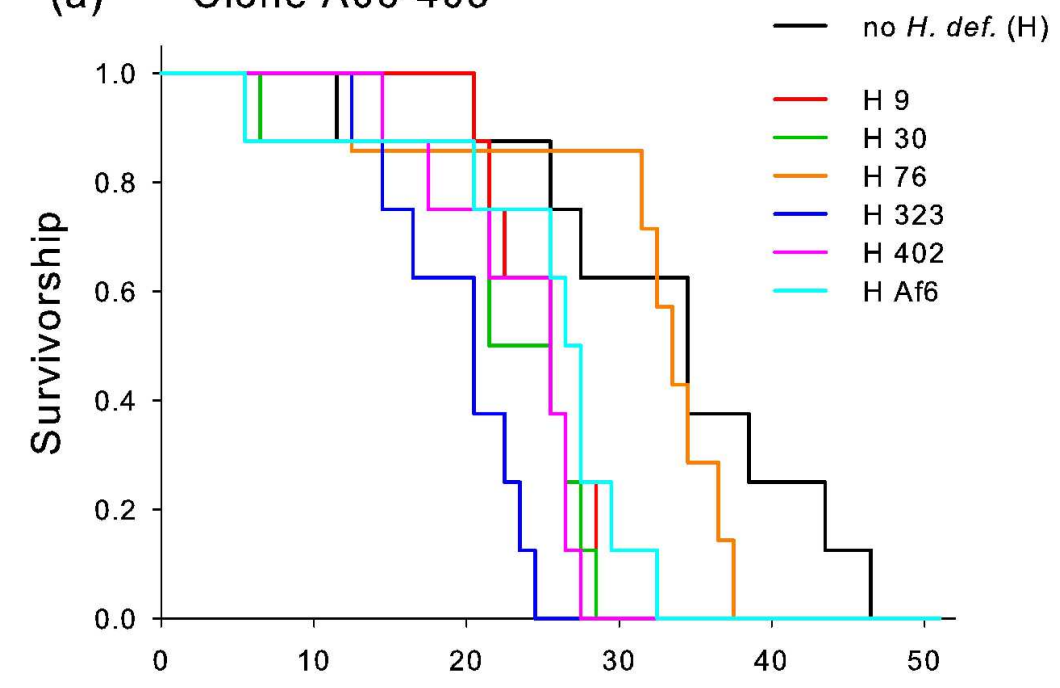

(b) Clone A06-407

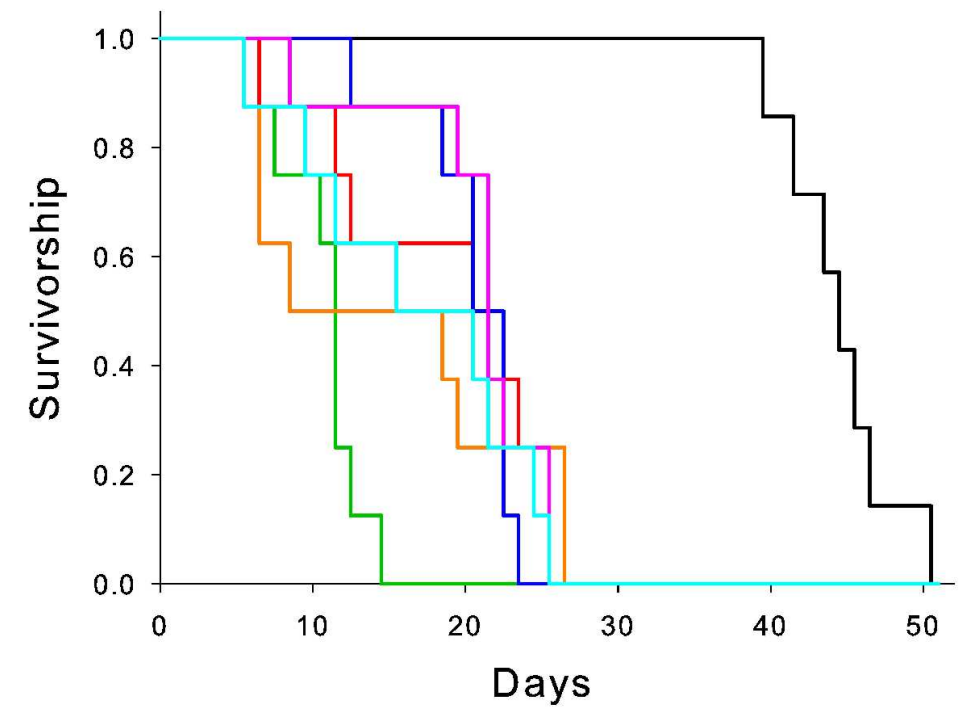

$166 \times 245 \mathrm{~mm}(600 \times 600 \mathrm{DPI})$ 